\title{
Ocorrência da hanseníase no Brasil e os principais métodos de diagnósticos laboratoriais
}

A hanseníase é uma doença infecciosa, de cunho crônico, ocasionada pelo Mycobacterium leprae. Essa actinobactéria bacilar possui afinidade com pele e nervos periféricos. É transmitida por meio do contato contínuo com pessoas multibacilares que não foram tratadas. A hanseníase é um agravo de relevância na saúde pública dos países em desenvolvimento. O Brasil está em segundo lugar na prevalência de casos novos no mundo, sendo que mais de $20 \%$ dos casos apresentam algum grau de incapacidade física já instalada. Este estudo visa identificar a ocorrência da hanseníase no Brasil e mostrar os principais exames laboratoriais existentes, dando ênfase à importância das ações de controle da doença e a aplicação do diagnóstico rápido e preciso. Foi realizada uma pesquisa literária, utilizando artigos e dissertações por meio dos sites, Scientific Electronic Library Online (SCIELO), Google Acadêmico e Biblioteca Virtual em Saúde (BVS). O levantamento de dados foi coletado através do site do Departamento de Informática do SUS (DATASUS) e do Portal da Saúde do SUS. Os últimos dados publicados mostram que em 2016, 25.218 novos casos de hanseníase foram detectados, com uma taxa de prevalência de 1,10 a cada 10.000 habitantes. 0 diagnóstico de hanseníase é basicamente clínico e epidemiológico, complementado, quando possível, com exame histopatológico e baciloscópico.

Palavras-chave: Hanseníase; Diagnóstico laboratorial; Mycobacterium leprae; Prevalência.

\section{Occurrence of leprosy in Brazil and the main methods of laboratory diagnosis}

\begin{abstract}
Leprosy is an infectious, chronic disease caused by Mycobacterium leprae. This bacillary actinobacterium has affinity with skin and periphera nerves. It is transmitted through continuous contact with multibacillary people who have not been treated. Leprosy is a major public health concern in developing countries. Brazil ranks second in the prevalence of new cases in the world, with more than $20 \%$ of cases having some degree of physical disability already installed. This study aims to identify the occurrence of leprosy in Brazil and show the main laboratory exams, emphasizing the importance of disease control actions and the application of rapid and accurate diagnosis. A literary research was carried out, using articles and dissertations through the Scientific Electronic Library Online (SCIELO), Google Scholar and Virtual Health Library (VHL). The data collection was collected through the website of the Department of Information Technology of SUS (DATASUS) and the Health Portal of SUS. The latest published data show that in 2016, 25,218 new cases of leprosy were detected, with a prevalence rate of 1.10 per 10,000 inhabitants. The diagnosis of leprosy is basically clinical and epidemiological, complemented, if possible, with histopathological and bacilloscopic examination.
\end{abstract}

Keywords: Leprosy; Laboratory diagnosis; Mycobacterium leprae; Prevalence.

Topic: Microbiologia

Reviewed anonymously in the process of blind peer.
Received: 11/12/2017

Approved: 22/12/2017
Mara Soares de Almeida Mota

Faculdade Guaraí, Brasi

http://lattes.cnpq.br/3061777057739833

http://orcid.org/0000-0002-2068-3481

mara.almeida06@gmail.com

Vanderlito Alves Vilanova

Faculdade Guaraí, Brasil

http://lattes.cnpq.br/0143278512192573

vanderlito.bio@gmail.com

Simone Pôssas Andrade

Faculdade Guaraí, Brasil

http://lattes.cnpq.br/0552128506796918

simone.andrade@iescfag.edu.br
Bruna Lira Barros Silva

Faculdade Guaraí, Brasil

http://lattes.cnpq.br/1592852846351586

brunabarros15@outlook.com

Darliana Oliveira Ferreira

Faculdade Guaraí, Brasil

http://lattes.cnpq.br/8924448974787850

darliana_pk@hotmail.com
Referencing this:

MOTA, M. S. A.; VILANOVA, V. A.; ANDRADE, S. P.; SILVA, B. L. B.; FERREIRA, D. O Ocorrência da hanseníase no Brasil e os principais métodos de diagnósticos laboratoriais. Scire Salutis, v.7, n.2, p.10-19, 2017. DOI: http://doi.org/10.6008/SPC2236-9600.2017.001.0002 


\section{INTRODUÇÃO}

Conhecida na antiguidade como lepra, a hanseníase é uma das mais antigas doenças registradas pela humanidade. Porém, apenas no final do século XIX foi que o médico Gerhard Herink Armauer Hansen, descobriu a Mycobacterium em material de lesões cutâneas. A hanseníase é uma enfermidade infectocontagiosa, crônica e ocasionada pelo Mycobacterium leprae, que acomete especialmente a pele e nervos periféricos. Apesar de possuir uma variedade de manifestações clinicas, a neuropatia periférica é a mais prevalente e responsável pelo potencial da doença em causar incapacidades e deformidades físicas. Quanto mais precocemente for o diagnóstico e o correto tratamento, maior a probabilidade de cura sem sequelas (MAEDA, 2011; SOUZA et al., 2013; CAIRES, 2013).

De acordo com Fracaroli et al. (2011), a transmissão da doença sucede por meio do contato contínuo com pessoas multibacilares que não foram tratadas e as fontes principais do bacilo são as mucosas das vias aéreas superiores. O diagnóstico é realizado através do exame clínico, complementado pelo cumprimento da anamnese, avaliação dermatológica a qual verifica as alterações ou lesões na pele e neurológica que analisa a presença de alteração de sensibilidade e motora e espessamento neural; e exame laboratorial, sendo o principal, por meio da baciloscopia, em que se observa o $M$. leprae diretamente nos esfregaços de raspados intradérmicos das lesões hansênicas ou de outros locais, como os lóbulos da orelha e/ou cotovelos (CAIRES, 2013).

A hanseníase é um agravo de relevância na saúde pública dos países em desenvolvimento. O Brasil está em segundo lugar na prevalência de casos novos no mundo, sendo que, mais de $20 \%$ dos casos apresentam algum grau de incapacidade física já instalada. Com isso, a hanseníase continua apresentando destaque perante as doenças negligenciadas (FRACAROLI et al., 2011; QUEIROZ, 2015). É importante também ressaltar que o preconceito e a atenuação da qualidade de vida dos portadores de hanseníase evidenciam ainda mais com a existência das incapacidades físicas consequentes dos estados reacionais, visto que esses elementos afetam diretamente nas condições físicas, sociais, econômicas e psicológicas de seus portadores (QUEIROZ, 2015).

Em razão da falta de conhecimento de todos os critérios clínicos e laboratoriais da hanseníase, como a ausência de um modelo experimental procedente e a inviabilidade de se cultivar o $M$. leprae, fica difícil o entendimento dos quesitos basilares da enfermidade, como a transmissão, a afinidade neurológica da micobactéria e a susceptibilidade de algumas pessoas para a evolução de formas mais graves ou mais bacilíferas. Por conseguinte, a análise epidemiológica da hanseníase continua sendo um recurso fundamental para o entendimento do seu ciclo de transmissão (CUNHA, 2012).

Diante desses dados e sendo a hanseníase uma doença antiga e que ainda é evidente diante das doenças negligenciadas e com grande ocorrência no Brasil, é importante ressaltar os índices da ocorrência desta enfermidade no Brasil, visando a aplicação de medidas de controle, tratamento e conscientização da população que vivem nas áreas endêmicas, além de pesquisas epidemiológicas como ferramenta essencial para a minimização da manifestação dessa doença no país. Outro fator determinante é que o prévio e correto 
diagnóstico é fundamental para o controle e eliminação da doença, tendo em vista que, em muitos casos a hanseníase passa despercebida. Desta forma, esse estudo tem como objetivo identificar a ocorrência da hanseníase no Brasil e mostrar os principais exames laboratoriais existentes, a importância das ações de controle da doença e o diagnóstico rápido e preciso.

\section{METODOLOGIA}

Este estudo foi desenvolvido com base em uma pesquisa literária e um levantamento de dados realizado no site do Departamento de Informática do SUS (DATASUS) e no Portal da Saúde do SUS, no período de janeiro 2017 a outubro de 2017. Para descrição desta elaboração, foram utilizados dissertações e artigos, empregando as seguintes palavras-chave: 'Hanseníase'; 'diagnóstico laboratorial'; 'prevalência'; e 'Mycobacterium leprae'. Os artigos foram encontrados em sites como Scientific Electronic Library Online (SCIELO), Google Acadêmico e Biblioteca Virtual em Saúde (BVS). As informações obtidas correspondem ao período de 2008 a 2017.

\section{DISCUSSÃO TEÓRICA}

\section{Hanseníase}

A hanseníase é uma enfermidade insidiosa, que acomete inicialmente o Sistema Nervoso Periférico, sendo comprovado por meio das manifestações clínicas, imunológicas e patológicas dos portadores. Em virtude dos aspectos bioepidemiológicos que ocasionam diversas manifestações clínicas e complicações, um diagnóstico fidedigno da doença, assim como sua adequada classificação, são exigências fundamentais para a segurança de um tratamento apropriado. Entretanto, detectar o Mycobacterium leprae no indivíduo é bastante dificultoso, de maneira que são essenciais diversos parâmetros clínicos e laboratoriais, pois não há um exame exclusivo e fidedigno para o diagnóstico da doença (TEIXEIRA, 2008).

A hanseníase pode ser classificada de duas formas: a paucibacilar, forma em que o indivíduo possui poucos bacilos. Essa classificação compreende a forma indeterminada ou inicial; e a tuberculoide polar ou autolimitada, a outra forma de classificação da hanseníase é a multibacilar, que compreende as formas dimorfa e virchowiana, onde os portadores possuem uma maior carga bacilar, pois o sistema imune não possui a capacidade de eliminar os bacilos. Desta forma, os pacientes com hanseníase paucibacilares possuem poucas lesões de pele, e eventualmente afeta troncos nervosos; já os multibacilares possuem múltiplas lesões e acometimento extenso de nervos (BARRETO, 2014).

O aparecimento dos sintomas da hanseníase resulta mais da resposta imunológica do portador ao Mycobacterium leprae do que da capacidade de multiplicação dos bacilos. São antecedidas por período de incubação prolongado, entre 2 e 10 anos (LASTÓRIA et al., 2012). A Hanseníase tem cura, mas para isso o diagnóstico tem que ser rápido e precoce. Algumas dificuldades podem ser apresentadas e muitas vezes o quadro clínico é confundido com o de diversas doenças dermatológicas, sendo o diagnóstico tardio um problema grave associado à doença. 
A demora no início do tratamento em decorrência do atraso no diagnóstico pode causar sequelas físicas, deixando o paciente com limitações em suas atividades da vida diária, além da doença já poder ter acometido a parte das vísceras e os nervos (SIMON, 2013). A magnitude da doença não é só analisada pela quantidade de doentes existentes, ou pela sua contagiosidade, mas devido as incapacidades que produz, questões psicossociais e pelo extenso espaço de tempo do tratamento (ARANTES et al., 2010).

\section{Diagnóstico Laboratorial da Hanseníase}

O diagnóstico na fase inicial da hanseníase permite auxiliar na interrupção do ciclo da transmissão da doença, além de intervir na redução ou impedir, a incapacidade nos pacientes (SALTARELI et al., 2016). Em grande parte dos estados brasileiros, ainda é tardio o diagnóstico da hanseníase: aproximadamente um ano e meio a dois anos depois do surgimento dos sintomas. A procura tardia de atendimento nos serviços de saúde, a ausência de informações a respeito das manifestações clínicas, a dificuldade das pessoas em encontrar serviços, atendimento ou profissionais qualificados para identificar a doença, são considerados elementos que levam a demora no diagnóstico (ARANTES et al., 2010).

O ambulatório Souza-Araújo é uma unidade de assistência do Laboratório de Hanseníase do Instituto Oswaldo Cruz e o único acreditado internacionalmente. Seu quantitativo de atendimento revela um dos principais desafios no combate à doença no país, que é o correto diagnóstico. No ano de 2014, 805 usuários procuraram o ambulatório com hipótese de hanseníase. Mas desses, somente 115 verdadeiramente eram portadores da doença, ou seja, $14,28 \%$ dos indivíduos. A maioria deles foram encaminhados por outros serviços de saúde, o que significa que não haviam passado por um procedimento eficaz de diagnóstico (KRAPP, 2015).

Dentre os diagnósticos laboratoriais da hanseníase estão a ultrassonografia e ressonância magnética, que auxiliam na identificação do tipo neural pura e neurite; a eletroneuromiografia é utilizada no rastreamento das reações; a baciloscopia, intradermorreação de Mitsuda e histopatologia, que comumente possibilitam diagnosticar e relacionar a forma clínica; e a sorologia, reação de imunistoquímica e reação em cadeia da polimerase (PCR) são métodos empregados basicamente em pesquisas (LASTÓRIA et al., 2012).

\section{Baciloscopia}

O exame baciloscópico do raspado intradérmico é o método mais utilizado pela facilidade na execução, pouco invasivo e de baixo custo. Ele é solicitado pelo médico para diagnóstico diferencial, ou com suspeita de recidiva da doença (BRASIL, 2010). A baciloscopia compreende raspado dérmico de lesão, lóbulos das orelhas e cotovelos, realizada através do método de Ziehl-Neelsen, a qual avalia os índices baciloscópico e morfológico.

O índice baciloscópico evidencia o número de bacilos em uma escala logarítmica entre 0 e 6+, onde os multibacilares é positiva, favorecendo no diagnóstico; os paucibacilares é regularmente negativa. 0 percentual de bacilos íntegros em relação ao total dos bacilos examinados constata viabilidade bacilar. Os 
íntegros, ou seja, viáveis, evidenciam-se totalmente corados em vermelho e manifestam nos casos sem tratamento ou recidivas. Os fragmentados destacam pequenas falhas na parede e os granulosos, grandes falhas revelando, respectivamente, fragmentos ou pontos corados em vermelho. São inviáveis e observados em pacientes tratados (LASTÓRIA et al., 2012; BARRETO, 2014).

\section{Histopatologia}

A histopatologia é útil para realizar diagnóstico na classificação da doença em situações em que a clínica suscita dúvida. Consiste no estudo da pele lesionada durante e depois da lesão. É um método um pouco invasivo e caro, sendo pouco usado. Observam-se as diferentes fases da hanseníase, como a indeterminada, tuberculoide e a virchowiana, que demonstram diferentes formas na pele hanseniana (BRASIL, 2010; OBADIA et al., 2011). A classificação histopatológica, pode revelar características das fases da doença em diferentes lesões de um mesmo paciente ou, raramente, alterações de diferentes fases em uma mesma amostra. O exame histopatológico não deve ser considerado como padrão ouro para o diagnóstico da doença, sendo complementar às características clínicas (OBADIA et al., 2011).

\section{Reação de Mitsuda}

$\mathrm{Na}$ intradermorreação de Mitsuda, injeta-se na derme o antígeno lepromina. O aparecimento de pápula igual ou maior que $5 \mathrm{~mm}$, passados quatro semanas, revela positividade. Demonstra o grau de imunidade celular, participando na classificação da forma clínica, todavia, não faz diagnóstico. É positiva em pacientes tuberculoides, onde há boa resposta imunocelular negativa nos virchowianos, em que essa resposta é deficiente, e evidencia graus variáveis de positividade nos dimorfos (LASTÓRIA et al., 2012).

\section{Sorologia}

O glicolipídeo fenólico-1, um dos antígenos específico do bacilo da hanseníase, está sendo um dos mais utilizados para pesquisa sorológica de anticorpos antiMycobacterium leprae, Através de ensaio imunoenzimático, ocorre a estimulação da produção de anticorpos IgM. O aparecimento desses anticorpos representa a carga bacilar, apresentando títulos aumentados nos multibacilares e diminuídos ou ausentes nos paucibacilares. No acompanhamento da terapia, a redução dos anticorpos acompanha o clearance do antígeno, ao passo que a persistência pode caracterizar a resistência terapêutica; já o aumento em pessoas tratadas pode significar recidiva. A sorologia também possibilita detectar pessoas com infecção assintomática (LASTÓRIA et al., 2012).

\section{Ocorrência da hanseníase no Brasil}

Através da incidência é possível avaliar o potencial de transmissão da doença e determinar as consequências das execuções que estão sendo implantadas para minimização da enfermidade. Por meio dos resultados é possível identificar os impactos das ações realizadas no decorrer do tempo e a necessidade de 
reforçá-las, instituindo atuações como publicação sobre a doença, os diversos diagnósticos e tratamento, campanhas regulares para avaliar as manchas nos indivíduos, investigação ativa nos domicílios pelos agentes comunitários de saúde e toda Equipe de Saúde da Família e a participação intensa dos usuários (SANTOS et al., 2016). Em 2012, foram registrados mundialmente cerca de 233 mil novos casos da doença, sendo 17\% de todos esses casos registrados no continente americano. 93\% desses, ocorrem no Brasil. (MONTEIRO et al., 2015)

Nos últimos anos, no Brasil, a detecção de novos casos da hanseníase decaiu. Houve uma redução de 42,2\% no número de novos casos, passando de 43.642 em 2006 para 25.218 em 2016. Também é importante resaltar que o número de unidades de saúde com pacientes em tratamento, houve um acréscimo, em 2006 foi registrado 6.988 unidades com pacientes em tratamento, já em 2016 os dados confirmarm 8.213. A porcentagem de avaliados quanto ao grau de incapacidade física (GIF) no diagnóstico foi de $86,6 \%$ em 2006, e de $87,3 \%$ em 20016 , ou seja, houve um leve aumento nesses casos específicos. As regiões com maior índice de detecção geral de hanseníase por 100 mil habiantes no ano de 2016 foram: a Região Centro-Oeste, com uma taxa de 30,02; as Regiões Norte e Nordeste, com 28,7 e 19,3, respectivamente. A Região Sul é a que aprensetou menor taxa, sendo de 2,84. A tabela 1 apresenta a taxa de deteç̧ão geral de hanseníase por 100 mil habitantes no anos de 2006 a 2016 (BRASIL, 2016).

Tabela 1: Detecção geral da hanseníase por 100 mil habitantes no Brasil de 2006 a 2016.

\begin{tabular}{|l|l|l|l|l|l|l|l|l|l|l|l|}
\hline Regiões & $\mathbf{2 0 0 6}$ & $\mathbf{2 0 0 7}$ & $\mathbf{2 0 0 8}$ & $\mathbf{2 0 0 9}$ & $\mathbf{2 0 1 0}$ & $\mathbf{2 0 1 1}$ & $\mathbf{2 0 1 2}$ & $\mathbf{2 0 1 3}$ & $\mathbf{2 0 1 4}$ & $\mathbf{2 0 1 5}$ & $\mathbf{2 0 1 6}$ \\
\hline Brasil & $\mathbf{2 3 . 3 7}$ & $\mathbf{2 1 , 1 9}$ & $\mathbf{2 0 , 5 9}$ & $\mathbf{1 9 , 6 4}$ & $\mathbf{1 8 , 2 2}$ & $\mathbf{1 7 , 6 5}$ & $\mathbf{1 7 , 1 7}$ & $\mathbf{1 5 , 4 4}$ & $\mathbf{1 5 , 3 2}$ & $\mathbf{1 4 , 0 7}$ & $\mathbf{1 2 , 2 3}$ \\
\hline Região Norte & 61,12 & 54,34 & 54,69 & 49,94 & 42,73 & 42,65 & 42,24 & 35,89 & 35,41 & 29,65 & $\mathbf{2 8 , 7}$ \\
\hline Nordeste & 32,28 & 31,75 & 30,04 & $\mathbf{2 8 , 8}$ & 27,73 & 26,08 & $\mathbf{2 5 , 7 8}$ & 23,79 & 24,07 & 22,72 & 19,3 \\
\hline Região Sudeste & 10,53 & 9,76 & 8,78 & 8,42 & 7,66 & 7,42 & 6,6 & 5,58 & 5,3 & 4,71 & 4,17 \\
\hline Região Sul & 6,99 & 6,44 & 6,05 & 5,54 & 5,19 & 4,99 & 4,83 & 4,08 & 3,57 & 3,49 & 2,84 \\
\hline Região Centro-Oeste & 56,6 & 41,19 & 44,64 & 44,28 & 41,29 & 40,4 & 40,04 & 38,59 & 38,62 & 44,3 & 30,02 \\
\hline
\end{tabular}

Um fator importante a se destacar é que, mesmo com as ações desenvolvidas no Brasil para o controle da hanseníase nos últimos anos, casos em menores de 15 anos ainda são diagnosticados no país, os quais evidenciam focos de infecção ativos e transmissão recente da enfermidade. Em 2016, a quantidade de novos casos detectados nesta faixa etária foi 1.696, representando $7 \%$ do total de casos novos do país. 0 diagnóstico de casos com Grau 2 de Incapacidade Física (GIF2) aponta para o diagnóstico demorado da doença. O percentual de casos novos da doença em 2016, diagnosticada com GIF2 foi de 7,9\%, dentre os analisados para este indicador no momento do diagnóstico (BRASIL, 2017).

Apesar da redução no número de casos da hanseníase nos últimos dez anos, pesquisadores da Fundação Oswaldo Cruz afirmam que a hanseníase é uma doença invisível, pois não é um agravo muito divulgado e pouco estudado pela saúde coletiva. Devido a dada irrelevância, o infectato tenta escondê-la. Por dificilmente causar mortes, suas estatísticas não são alarmantes; logo, é uma enfermidade que não causa incômodo a ninguém, a não ser aos próprios portadores. Foi resaltado também que o diagnóstico da hanseníase tem sido menosprezado pela própria classe médica, pois, nem sempre é o paciente que demora 
a solicitar um atendimento, mas a falta de profissionais qualificados que não fazem a correta identificação da doença, e cada pessoa não diagnósticada, vai infectar pelo menos outros dois indivíduos (KRAPP, 2015).

Uma análise realizada, a respeito do perfil clínico e epidemiológico de pacientes em reação hansênica, com 61 pacientes, em um município do Nordeste brasileiro, revelou que essas reações acometem em maior número as pessoas que dispõem de baixas condições socioeconômicas, o que confirmou a associação entre pobreza e hanseníase. Foi evidenciado também que a maioria dos pacientes apresentavam algum tipo de incapacidade física quando realizaram o diagnóstico da hanseníase, constatando então, que neste município, o diagnóstico da doença tem sido tardio, o qual ocasiona uma importante barreira no controle da hanseníase e prevenção de incapacidades (QUEIROZ et al., 2015).

Um estudo realizado no município de Tucano/BA, entre 2002 e 2012 foram notificados 91 casos de hanseníase, com uma média de 8,3 casos por ano. A taxa de incidência variou de 3,8 casos em 2002 para 41,6 em 2012 por 100.000 habitantes. A análise dos resultados aponta que existe uma persistência da transmissão ativa do Mycobacterium leprae e uma identificação tardia da doença, com padrões de hiperendemicidade na região do munícipio (SANTOS, 2016).

A prevalência da hanseníase nos últimos dez anos nas regiões do Brasil são persistentes, com uma tendência minima de redução e oscilações entre os anos de 2006 a 2016, desses, os anos de 2008 e 2009 foram os que apresentaram maior predomínio da doença. Em 2006 o Brasil tinha uma taxa 1,41 para cada 10.000 habitantes, em contrapartida em 2016 a incidência foi de 1,10, ou seja, uma redução de $22 \%$ na prevalência da doença no Brasil. A Região Centro-Oeste é a que apresenta a maior taxa de prevalência desde o ano de 2009 até 2016, sendo que de 2006 a 2009 era a Região Norte que apresentava maior prevalência. As regiões com menor prevalência, em 2016, compreende o Sul e Sudeste, com 0,25 e 0,36 respectivamente por 10.000 habitantes. Nas regiões Norte e Nordeste, em 2016, houve um aumento dessa taxa em relação ao ano de 2015. A tabela 2 apresenta a taxa de prevalência da hanseníase de 2006 a 2016 nas regiões do Brasil (BRASIL, 2016).

Tabela 2: Taxa de prevalência de hanseníase por 10.000 habitantes de 2006 a 2016.

\begin{tabular}{|l|l|l|l|l|l|l|l|l|l|l|l|}
\hline Regiões & $\mathbf{2 0 0 6}$ & $\mathbf{2 0 0 7}$ & $\mathbf{2 0 0 8}$ & $\mathbf{2 0 0 9}$ & $\mathbf{2 0 1 0}$ & $\mathbf{2 0 1 1}$ & $\mathbf{2 0 1 2}$ & $\mathbf{2 0 1 3}$ & $\mathbf{2 0 1 4}$ & $\mathbf{2 0 1 5}$ & $\mathbf{2 0 1 6}$ \\
\hline Brasil & $\mathbf{1 , 4 1}$ & $\mathbf{2 , 1 1}$ & $\mathbf{2 , 0 6}$ & $\mathbf{1 , 9 9}$ & $\mathbf{1 , 5 6}$ & $\mathbf{1 , 5 4}$ & $\mathbf{1 , 5 1}$ & $\mathbf{1 , 4 2}$ & $\mathbf{1 , 2 7}$ & $\mathbf{1 , 0 1}$ & $\mathbf{1 , 1 0}$ \\
\hline Região Norte & 3,78 & 5,43 & 5,42 & 5,02 & 3,47 & 3,49 & 3,43 & 3,07 & 2,62 & 2,00 & 2,37 \\
\hline Nordeste & 1,93 & 3,15 & 3,08 & 2,98 & 2,46 & 2,35 & 2,31 & 2,22 & 1,91 & 1,58 & 1,77 \\
\hline Região Sudeste & 0,65 & 0,97 & 0,86 & 0,75 & 0,62 & 0,61 & 0,57 & 0,48 & 0,42 & 0,34 & 0,36 \\
\hline Região Sul & 0,51 & 0,65 & 0,51 & 0,50 & 0,43 & 0,44 & 0,44 & 0,37 & 0,31 & 0,29 & 0,25 \\
\hline Região Centro-Oeste & 3,12 & 4,06 & 4,55 & 5,04 & 3,60 & 3,75 & 3,73 & 3,80 & 3,93 & 3,49 & 2,91 \\
\hline
\end{tabular}

Nereu Mansano, representante do Conselho Nacional de Secretários de Saúde (CONASS), afirma que a procura por casos de hanseníase deve ser prioridade em todos os estados do país, inclusive onde a prevalência seja reduzida. Ele enfatiza que é necessário estar atento aos municípios silenciosos, em que existam áreas de alta prevalência, porém com municípios que não apresentam casos. É nesses locais que é preciso estar alerta para que casos não sejam diagnosticados tardiamente (BRASIL, 2017). 
No ano de 2015, o estado do Mato Grosso apresentou a maior taxa de prevalência, estando classificado em alto risco. $O$ estado do Tocantins ocupa o segundo lugar na taxa de prevalência, seguido pelo Maranhão, sendo os dois classificados em risco médio. O estado com a menor taxa de prevalência é o Rio Grande do Sul, seguido de Santa Catarina e São Paulo, os quais estão em baixo risco na prevalência da hanseníase. (BRASIL, 2015; BRASIL, 2016). 0 gráfico 1 apresenta a taxa de prevalência da hanseníase nos estados brasileiros com base no último resultado apresentando no site do Departamento de Informática do SUS (DATASUS), que faz referência ao ano de 2015.

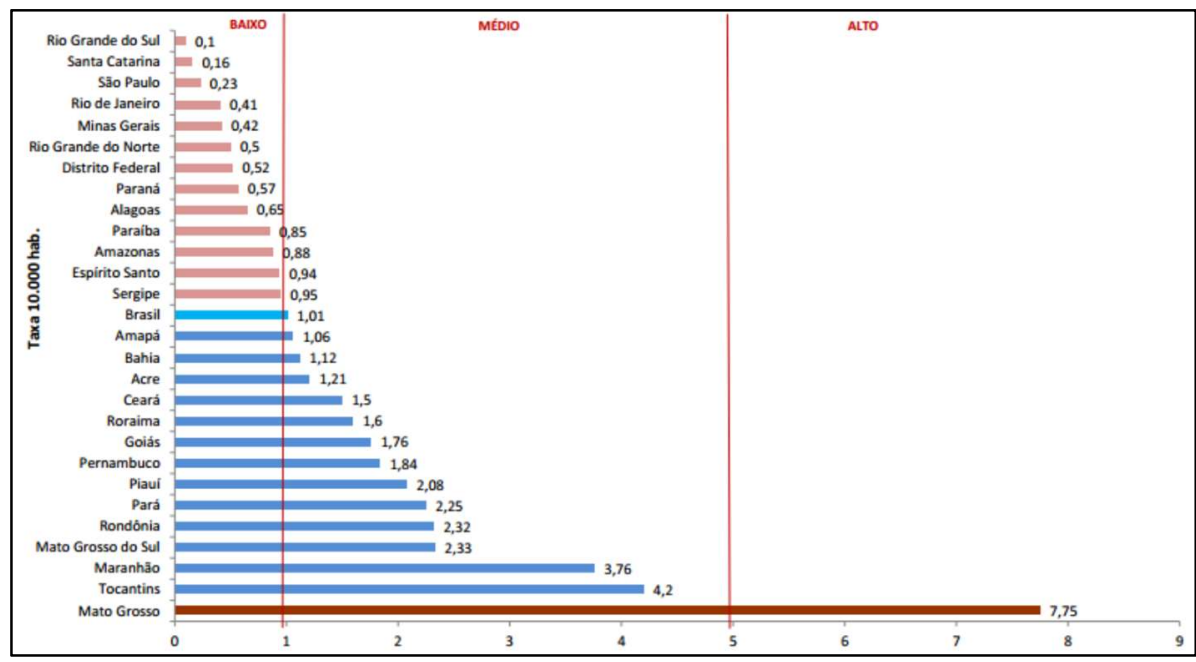

Gráfico 1: Taxa de prevalência da hanseníase por estados do Brasil em 2015. Fonte: Brasil (2015).

Uma pesquisa realizada sobre as tendências da hanseníase no estado do Tocantins, no périodo entre 2001 a 2012, revela a persistência da transmissão ativa e diagnóstico tardio da doença, com critérios de hiperendemicidade em todo o território. A significância e a tendência da hanseníase expostas no período analisado indicam que, isoladamente, as ações de controle do município, com a integral cobertura para o diagnóstico e tratamento da doença pela atenção primária, não é o bastante para a obtenção da meta de eliminação. Outros fatores que determinam a persistência da doença, sãos os processos migratórios de pessoas dos municípios vizinhos classificados como hiperendêmicos, tal como o Pará, Maranhão e Mato Grosso, e limitações referentes ao preenchimento das notificações através dos profissionais de saúde (MONTEIRO et al., 2015).

No Brasil, a hanseníase é uma das primazias do Ministério da Saúde. No entanto, mesmo que as estratégias atuais para o controle e eliminação da doença, tenham alcançado resultados positivos, ainda são insuficientes. A maior dificuldade reside na execução insatisfatória em nível municipal, pois para a melhor efetividade do controle e eliminação da doença, é preciso uma descentralização efetiva do diagnóstico e tratamento, por meio de métodos de diagnósticos e terapêuticos próximo a residência do paciente, para que a doença seja detectada em sua fase inicial, e que o tratamento seja imediato, evitando também o abandono do tratamento (SALTARELI et al., 2016).

A erradicação da hanseníase não está sendo fácil de ser efetivada em algumas regiões, em consequência da complexidade da doença. O bacilo Mycobacterium leprae é classificado como de baixa 
patogenicidade, dado que, somente um reduzido número de indivíduos possivelmente infectados desenvolve a doença. Ademais, não existe um conhecimento concreto, que se em um determinado momento entre o contato com o bacilo e a enfermidade, o portador assintomático se torna "bacilífero" e atua no decurso da transmissão da hanseníase (CUNHA et al., 2012)

No Brasil, é preciso fortalecer as ações de vigilância local da hanseníase direcionadas principalmente à melhor efetividade no diagnóstico e tratamento precoce da doença, sobretudo nas regiões que manifestam maior concentração de casos no país. Outrossim, é essencial o aprimoramento contínuo dos sistemas de informação, mecanismo esse indispensável para assegurar a apropriada supervisão da situação epidemiológica da hanseníase no Brasil e para auxiliar na finalidade de erradicação da doença como problema de saúde pública (SILVA et al., 2016).

Em junho de 2017, a Secretaria de Vigilância em Saúde (SVS), lançou o projeto 'Abordagens inovadoras para intensificar esforços para um Brasil livre de hanseníase', cuja finalidade consiste em diminuir a carga de hanseníase em 20 municípios dos estados de Mato Grosso, Maranhão, Pará, Tocantins, Pernambuco e Piauí. Esse projeto terá duração de 3 anos entre 2017 e 2019. Os objetivos para concretização do projeto incluem, aumentar as atuações de detecção precoce de casos de hanseníase; providenciar a capacitação dos profissionais de saúde na Hanseníase Clínica e em Prevenção de Incapacidades (PI) na esfera da atenção primária à saúde; diminuir a quantidade de novos casos da doença com Grau 2 de incapacidade física (GIF2); além de propiciar a atenuação do estigma e da discriminação contra os portadores da hanseníase na sociedade e conceder a população conhecimentos sobre as questões relacionadas à enfermidade (BRASIL, 2017).

\section{CONCLUSÕES}

Por meio deste estudo, conclui-se que a ocorrência da hanseníase ainda é persistente no Brasil, embora há uma boa abrangência dos serviços de saúde nos últimos 10 anos. Os resultados indicam que, além das medidas aplicadas através do programa de controle da doença, são necessários novos recursos de pesquisa para um conhecimento mais amplo a respeito dos mecanismos de transmissão da Mycobacterium leprae, como também identificar as regiões de maior risco e suas fontes potenciais de infecção, e com isso oferecer subsídios às medidas de controle nos estados. Esses dados reforçam substanciar o dever de dar maior atenção à hanseníase como condição crônica agregada de fato na rede de atenção do Sistema Único de Saúde. Para mais, é importante que realize uma revisão e revigoramento das ações de controle dos programas estaduais e municipais para que estas medidas sejam elencadas na realidade das diversas regiões e contextos da doença no Brasil.

É evidente que a resolução dos problemas relacionados a persistência da hanseníase nos estados brasileiros, exigem ações que permitam o diagnóstico precoce, tratamento e cura, que são fundamentais para a erradicação da doença, também é necessário investir em educação continuada para os profissionais 
da saúde, com a finalidade de que haja um atendimento e efetivo acompanhamento dos pacientes enquanto durar o tratamento e até após a cura.

\section{REFERÊNCIAS}

ARANTES, C. K.; GARCIA, M. L. R.; FILIPE, M. S.; NARDI, S. M. R.; PASCOAL, V. D.. Avaliação dos serviços de saúde em relação ao diagnóstico precoce da hanseníase. Epidemiologia e Serviços de Saúde, v.19, n.2, p.155-164, 2010.

BARRETO, J. A.. Diagnóstico laboratorial da hanseníase: indicações e limitações. In: ALVES, E. D.; FERREIRA, T. L.; FERREIRA, I. N.. Hanseníase: Avanços e desafios. Brasília, Universidade de Brasília, 2014. p.131-140.

BRASIL. Ministério da Saúde. Guia de procedimentos técnicos: baciloscopia em hanseníase. Brasília: Ministério da Saúde, 2010.

BRASIL. Ministério da Saúde. Hanseníase: situação epidemiológica. Brasília: Ministério da Saúde, 2016.

BRASIL. Ministério da Saúde. Informações de saúde, epidemiológicas e morbidade: banco de dados, casos de hanseníase desde 2001. Brasília: Ministério da Saúde, 2015.

BRASIL. Ministério da Saúde. Projeto lançado pela SVS intensifica esforços par redução da carga da hanseníase no Brasil. Brasília: Ministério da Saúde, 2017.

CAIRES, A. S.. Avaliação da função visual de pacientes com hanseníase em tratamento no centro de referências em doenças tropicais: CRDT em Macapá no Amapá. Dissertação (Mestrado em Ciências da Saúde) - Universidade Federal do Amapá, Macapá, 2013.

CUNHA, M. D.; SANTOS, R. S.; MATOS, H. J.; OLIVEIRA, M. L. W.. Aspectos epidemiológicos da hanseníase: uma abordagem espacial. Cadernos de Saúde Pública, Rio de Janeiro, v.28, n.6, p.1143-1153, 2012.

FRACAROLI, T. S.; MIRANDA, L. Q.; BRINGEL, D. M.; OBADIA, D. L.; DAXBACHER, E. L. R.. Importância da clínica no diagnóstico da hanseníase. Revista Hospital Universitário Pedro Ernesto, Rio de Janeiro, v.10, n.1, p.29-35, 2011.

KRAPP, J.. Hanseníase ainda é uma doença invisível, afirmam pesquisadores. Rio de Janeiro: Fundação Oswaldo Cruz, 2015.

LASTÓRIA, J. C.; ABREU, A. M. M.. Hanseníase: diagnóstico e tratamento. Diagnóstico e Tratamento, v.17, n.4, p.173-179, 2012.

MAEDA, S. M.. Hanseníase. Revista RBM Especial Dermatologia e Cosmiatria, v.68, p.19-22, 2011.
MONTEIRO, L. D.; MELO, F. R. M.; BRITO, A. L.; LIMA, M. S.; ALENCAR, C. H.; HEUKELBACH, J.. Tendências da hanseníase no Tocantins, um estado hiperendêmico do Norte do Brasil, 2001-2012. Caderno de Saúde Pública, Rio de Janeiro, v.31, n.5, p.971-980, 2015.

OBADIA, D. L.; VERARDINO, G.; ALVES M. F. G. S.. Hanseníase: correlação clínico-histopatológica. Revista Hospital Universitário Pedro Ernesto, Rio de Janeiro, v.10, n.1, p.20-23, 2011

QUEIROZ, T. A.; CARVALHO, F. P. B.; SIMPSON, C. A.; FERNANDES, A. C. L.; FIGUEIRÊDO, D. L. A.; KNACKFUSS, M. I.. Perfil clínico e epidemiológico de pacientes em reação hansênica. Revista Gaúcha de enfermagem, Porto Alegre, v.36, p.185-91, 2015. DOI: http://doi.org/10.1590/19831447.2015.esp. 57405

SALTARELI, R. M. F.; SEIXAS, D. H. T.. Limites e possibilidades na atenção ao portador de hanseníase no âmbito da estratégia saúde da família. Revista Atenção Primaria a Saúde, v.19, n.4, p.613-622, 2016.

SANTOS, A. D.; SANTOS, M. B.; BARRETO, A. S.; CARVALHO, D. S.; ALVES, J. A. B.; ARAÚJO, K. C. G. M.. Análise espacial e características epidemiológicas dos casos de hanseníase em área endêmica. Revista de Enfermagem UFPE online, Recife, v.10, n.5, p.4188-4197, 2016. DOI:

http://doi.org/10.5205/reuol.9284-81146-1SM.1005sup201604

SILVA, J. C. A.; RIBEIRO, M. D. A.; OLIVEIRA, S. B.. Avaliação do nível de informação sobre hanseníase dos agentes comunitários de saúde. Revista Brasileira de Promoção à Saúde, Fortaleza, v.29, n.3, p.364-370, 2016

SIMON, M. V.. Resposta imune a antígenos de Mycobacterium leprae e Mycobacterium tuberculosis na proteção e na patogênese da hanseníase. Tese (Doutorado em Ciências da Saúde) - Universidade Federal de Sergipe, Aracaju, 2013.

SOUZA, M. M.; NETTO, E. M.; FARIAS, M. C. A. D..

Ferramentas no diagnóstico da hanseníase: o convencional e as inovações. Revista RBM Especial Dermatologia e Cosmiatria, v.70, p.16-22, 2013.

TEIXEIRA, A. C.; CRUVINEL, D. L.; ROMA, F. R.; LUPPINO, L. F.; RESENDE, L. H. P; SOUSA, T.; BÜHRER-SÉKULA, S.; GOULART, I. M. B.. Avaliação da concordância entre exames clínicos e laboratoriais no diagnóstico da hanseníase. Revista da Sociedade Brasileira de Medicina Tropical, v.41 n.2, p.4855, 2008. 